\title{
Model Reference Adaptive Control for Perforated Mill
}

\author{
Xiu-Chun Zhao, Guo-Kai Xu, TaoZhang and Ping-Shu Ge
}

Department of Electromechanical \& Information Engineering, Dalian Nationalities University, Dalian, 116600, China

Keywords: Model reference adaptive control, Perforated mill, Disturbances and uncertainties

\begin{abstract}
This paper addresses a model reference adaptive controller which has simpler structure, fewer adjustable parameters, easier operation and accurate tracking capability even in the presence of external disturbances and uncertainties. The controller is applied into the perforated mill automatic speed control system and simulation results illustrate the practicality and effectiveness.
\end{abstract}

\section{Introduction}

Since Massachusetts Institute of Technology first proposed adaptive control technology in the late 1950s [1], many different forms adaptive control method has appeared [2-5], such as Dressber and Price, Pearson, who early proposed the design method [5]. These methods can not guarantee the system global asymptotic stability. Later, Monopoli researched an augmented error signal method, which design adaptive control law by Jacobi stability theory[6]. It can adjust the controller input and output parameters to gain the good performance and avoid adopting the differential of output signal. However, this method has too many control parameters and the structure is complex to implement applications. Therefore, reducing the adjustable parameters in the model reference adaptive controller and simplifying the controller structure is the key to the practical application of adaptive controller [7-9]. Moreover, there is a variety of interference signals inevitably in actual system. While Improve the system robustness, ensures the system stability even in the presence of external disturbances and uncertainties have become the urgent problem.

Electric drive system is one of the most successful areas in model reference adaptive control system application [10-12]. For example, C.Lascu and I.Boldea adopt adaptive control for motor supply drive system in the early application [11]. Model reference adaptive controller can design the reference model based on the system performance, and the adaptive controller can ensure the system tracking and stability by adjusting the controller parameters even in the presence of external disturbances and uncertainties. Perforated mill speed control system is mainly focused on controlling the drive electrical motor speed. According to the difference between the measured values of servo motor and set values, the precise speed control is achieved by control servo motor.

Consider above reasons, this paper addresses a practical model reference adaptive controller for perforated mill speed control system. The controller obtains the feedback gain from the filter derivative of control input, so the system has good control performance even in the presence of external disturbances and uncertainties. The controller has simple structure and fewer tuning parameters, which make the proposed reference model adaptive controller has the practical value.

\section{Perforated mill speed control system}

Perforated mill is generally applied into seamless steel pipe rolling equipment. The speed control plays a vital role on the production efficiency and product quality. Perforated mill speed control system is mainly focused on controlling the drive electrical motor speed. According to the difference between the measured values of servo motor and set values, the precise speed control is achieved by control servo motor. The control system adopts direct-current motor and silicon controlled rectifier technology and the motor current loop is used as controlled object. The system dynamic structure is shown in Fig.1. The system desired performance is the overshoot $M_{p}=10 \%$, rise time $_{t_{s}}=10$, so the reference model is selected as 
$\frac{y_{m}(s)}{r(s)}=\frac{20.89}{s^{2}+4.57 s+20.89}$

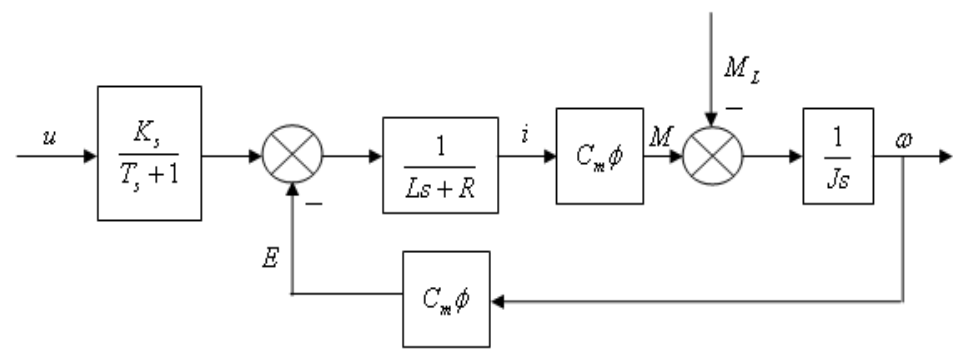

Fig.1 structure of perforated mill speed control

Notations:

$u$ : Armature voltage;

$K_{s}:$ Silicon controlled rectifier gain

$T_{s}$ : The control time of the silicon controlled rectifier;

$L:$ Armature Inductance

$R:$ Armature resistance;

$i$ : Armature current

$C_{m} \phi:$ Motor torque coefficient;

$M$ : Motor torque

$M_{L}$ : Motor load torque;

$J$ : Motor moment of inertia

\section{Model reference adaptive controller design}

The Model reference adaptive control principle is: According to the plant structure and control requirement, the reference model is designed to make output response can express the input commands expect. Then, the tracking error between the model output and the controlled object output by adjusting controller parameters, so that the tracking error converge to zero. That is, for any piecewise continuous uniformly bounded input function $r(t)$ and any initial conditions, the object control input can be found to make the tracking error between the controlled object and reference model gradually converge to zero.

The system reference model equation is depicted by input-output equations as:

. $A_{m}(p) Y_{m}(t)=B_{m}(p) r(t)$

Where, $A_{m}(p)=p^{n-h}+\sum_{i=0}^{n-h-1} a_{i} p^{i}, B_{m}(p)=\sum_{i=0}^{m_{2}} b_{i} p^{i} . m_{2} \leq n-h-1 . a_{i}, b_{i}$ are Known constant, $r$ and $Y_{m}$ are the reference model input and output respectively, $h$ is the order difference between controlled object and reference model, $p$ is differential operator in all polynomials, $p=d / d t$.

The corresponding controlled object is depicted by input-output equations as:

$A_{p}(p) Y_{p}(t)=B_{p}(p) u_{p}(t)$

Where, $A_{p}(p)=p^{n}+\sum_{i=0}^{n-1} \alpha p^{i}, B_{p}(p)=\sum_{i=0}^{m_{1}} \beta_{i} p^{i}, m_{1} \leq n-1$

$\alpha_{i}, \beta_{i}$ both are uncertain slow time-varying coefficients, $u_{p}$ and $Y_{p}$ are the controlled object input and output respectively, $B_{p}$ is Hurwitz polynomial.

The tracking error is $e(t)=Y_{m}(t)-Y_{p}(t)$

The structure of model reference adaptive controller is shown as fig.2. 
Suppose that:

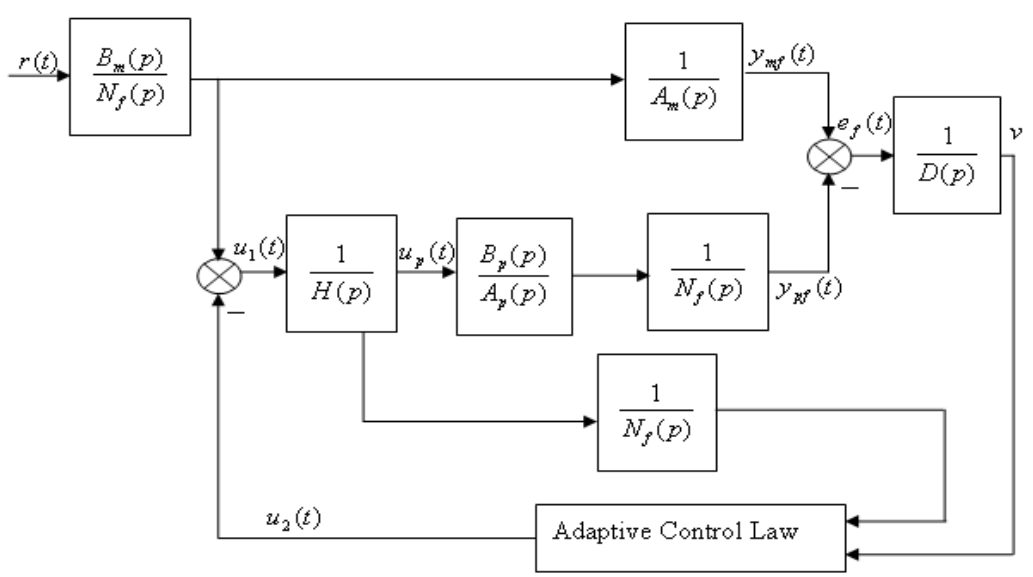

Fig.2 structure of model reference adaptive controller

$$
A_{p}(p)=A_{p}^{\prime}(p)+A_{m}(p), \quad A_{p}^{\prime}(p) Y_{p}(t)=M(p) u_{p}(t)
$$

Where, $A_{p}^{\prime}(p)=p^{n}+\sum_{i=0}^{n-1} \alpha_{i}^{\prime} p^{i}, M(p)=\sum_{i=0}^{m_{1}} m_{i} p^{i}, \alpha_{i}^{\prime}, m_{i}$ both are uncertain slow time-varying coefficients.

Combining (4) into (3), the controlled object can be expressed as follows:

$$
A_{p}^{\prime}(p) Y_{p}(t)+A_{m}(p) Y_{p}(t)=B_{p}(p) u_{p}(t)
$$

The tracking error $e(t)=Y_{m}(t)-Y_{p}(t)$ is introduced into (2)-(5), then

$$
A_{m}(p) e(t)=B_{m}(p) r(t)-B_{p}(p) u_{p}(t)+A_{p}^{\prime}(p) Y_{p}(t)
$$

Construct controller output as follows,

$$
\frac{B_{m}(p) r(t)}{N_{f}(p)}=u_{1}(t)+u_{2}(t), u_{1}(t)=H(p) u_{p}(t)
$$

Where, $1 / N_{f}(p)$ is state filter, $N_{f}(p)=\sum_{i=0}^{n-h-1} f_{i} p^{i}, H(p)=\sum_{i=0}^{h} m_{i} p^{i}, h_{i}$ and $f_{i}$ are constant.

Substituting (7) into (6), The error function can be described as,

$$
A_{m}(p) e_{f}=u_{2}(t)+\left[N_{f}(p) H(p)-B_{p}(p)+M(p)\right] u_{p f}(t)
$$

Where, $e_{f}=\frac{1}{N_{f}(p)} e, u_{p f}=\frac{1}{N_{f}(p)} u_{p}$

Substituting linear compensator $D(p)$, then

$v=\frac{D(p)}{A_{m}(p)}\left\{u_{2}(t)+\left[N_{f}(p) H(p)-B_{p}(p)+M(p)\right] u_{p f}(t)\right\}$

Adaptive control output $u_{2}(t)$ can be expressed as

$$
u_{2}(t)=\hat{K}(p) u_{p f}(t)
$$

Using (10) and (9) the generalized error equation can be further written as

$$
v=\frac{D(p)}{A_{m}(p)}\left\{\left[\hat{K}(p)+N_{f}(p) H(p)-B_{p}(p)+M(p)\right] u_{p f}(t)\right\}
$$

Where, $\hat{K}(p)=\sum_{i=0}^{m_{1}} k_{i} p^{i}$. 
If the appropriate adaptive update law for $k_{i}$ can be selected and meet the following conditions, we may easily prove the convergence of the tracking errors to zero and the system stability using Popov hyper-stability theory.

The conditions are:

Firstly, transfer function $D(p) / A_{m}(p)$ is strictly positive real,

Then the Proportional-integral adaptive law is defined as

$$
\frac{d}{d t} k_{i}=-k_{i 1} v p^{i} u_{p f}-k_{i 2} \frac{d}{d t}\left(k_{i 1} v p^{i} u_{p f}\right) \quad i=0, \quad 1, \quad \ldots, \quad n-1
$$

Where, $k_{i 1}$ and $k_{i 2}$ are the gain coefficients of the adaptive control law.

Finally, the system control input $u_{p}$ is

$$
u_{p}=\frac{1}{H(p)}\left[B_{m}(p) r_{f}+\sum_{i=0}^{n-1} k_{i} p^{i} u_{p f}\right]
$$

\section{Simulation results}

The controlled object is perforated mill speed control system which structure is shown in fig.1. Drive motor parameters are selected as $P_{e}=1500 \mathrm{~kW}, I_{e}=1720 \mathrm{~A}, R=0.0314 \Omega$, $L=0.003 \mathrm{H}, J=1547 \mathrm{~kg} / \mathrm{m} 2, K_{s}=152, T_{s}=1.67 \mathrm{~ms}, C_{m} \phi=29 \mathrm{Nm} / \mathrm{A}$, the maximum allowable value of the bound is 4 . In order to verify the effectiveness of the proposed model reference adaptive control, the reference model is selected as two-order function (1). The structure of model reference adaptive controller is shown in fig.2. The control gains used in the simulation were selected as $k_{0}=500, k_{1}=0.02, k_{2}=0.01$. Applying Popov hyper-stability theory and Matlab/simulation, simulation results are shown in Fig.3-4.

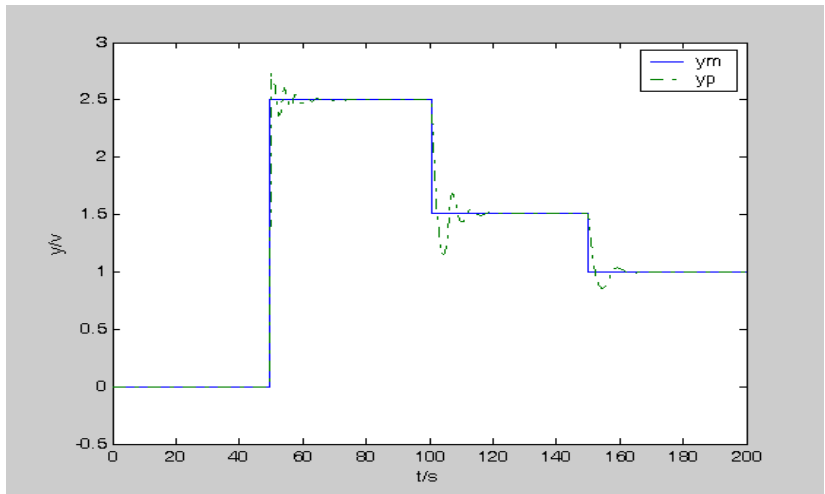

Fig.3 Output response of the reference model and controlled object

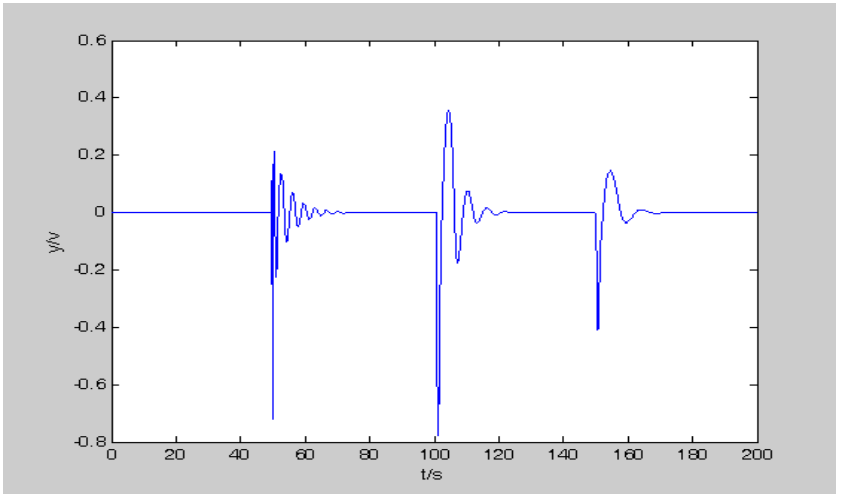

Fig.4 Tracking errors of the adaptive controller 


\section{Summary}

A model reference adaptive controller for perforated mill speed control system is presented. The controller obtains the feedback gain from the filter derivative of control input, so the system has good control performance even in the presence of external disturbances and uncertainties. Adaptive control law is based on the Popov hyper-stability theory, which makes the system have good tracking and stabilities. The controller has simple structure and fewer tuning parameters, which make the proposed reference model adaptive controller has the practical value. The effectiveness of the proposed controller was verified by simulation.

\section{Acknowledgments}

This work was supported by Fundamental Research Funds for the Central Universities, Foundation of Liaoning Educational Committee (L2010093).

\section{References}

[1] Wu shichang, Wu zhongqiang: Adaptive Control (Beijing: Mechanical Industry Press 2005)

[2] J. H. Kim. Delay and its time-derivative dependent robust stability of time delayed linear systems with uncertainty, IEEE Transactions on Automatic Control, Vol.46, No.5 (2001), p.789-792.

[3] J. Cao, J. Wang. Delay dependent robust stability of uncertain nonlinear systems with time delay, Applied Mathematics and Computation, Vol.154, No.2(2004), p.289-297.

[4] Y. He, Q. Wang, L. Xie, etal. Further improvement of free-weighting matrices technique for systems with time-varying delay, IEEE Transactions on Automatic Control, Vol.52, No.2(2007), p.293-299.

[5] Li qingquan. Adaptive Control System Theory, Design and Aapplication(Beijing: Science Press 1990)

[6] Wu zhongqiang, Piao chunjun. Overview of Model reference adaptive control theory, Information technology, Vol.25, No.7( 2000), p.33-34.

[7] Zhang pengfei, Peng weidong. Control of Induction Motor Based on Model Reference Adaptive System, Modern Electronics Technique, Vol.34, No.1(2011), p.196-199.

[8] Xu guokai, Chu zhenyou, WU Shichang. The adaptive controller design of sport system, Beijing: Chinese technology press, 2004.

[9] Liu xing, Liu xiao. The development and application of self-adaptive control system, Academic forum, Vol.53, No.9(2011), p.61-63.

[10] Zhao xiuchun, Xu guokai. Adaptive Control of Double Housing Planer, Proceedings of the 27th Chinese control conference, Vol.20, No.7(2008), p.20-23.

[11] C.Lascu, I.Boldea, and F.Blaabjerg. A modified direct torque control for induction motor sensorless drive," IEEE Trans. Ind, No.4(2000), p.122-130.

[12] Mohamed Boukattaya,Tarak Damak, Mohamed Jallouli. "Robust Adaptive Control for Mobile Manipulators,"International Journal of Automation and Computing, No.2(2011), p. 8-13. 